\title{
Self-Similar Constructions in Smooth Dynamics: Rigidity, Smoothness and Dimension
}

\author{
Jean-Marc Gambaudo ${ }^{1}$ and Charles Tresser $^{2}$ \\ 1 INLN, Faculté des Sciences, Parc Valrose, F-06108 Nice Cedex 02, France \\ 2 I.B.M. Thomas J. Watson Research Center, Po Box 218, Yorktown Heights, NY 10598, USA
}

Received September 19, 1990

\begin{abstract}
In the first part of this paper, for each $d \geq 2$, we construct diffeomorphisms of the $d$-dimensional ball which have zero entropy, one periodic orbit with period $2^{n}$ for each $n \geq 0$, no other periodic orbits, and a single invariant Cantor set which has a continuum of possible but, in any case, simple geometric structures. These diffeomorphisms are $C^{r(d)}$-smooth, where $r(d)$ is a strictly increasing function of $d$, which goes to infinity with $d$. The second part contains a more general result about smooth maps obtained by an infinite sequence of surgeries, and further particular cases.
\end{abstract}

\section{General Introduction}

This paper contains two parts.

- In the first part, we show how a few straightforward ideas combine to give simple smooth maps at the accumulation of cascades of period doubling bifurcations, with a smoothness which gets improved when increasing the dimension (Theorem 1). This has the following consequence:

\section{The minimal smoothness required to hope for universality at the accumulation of period doubling bifurcations increases with the dimension.}

- The second part begins by a formulation of a general result, our Theorem 2 , which only needs obvious changes to the specific arguments used in the proof of Theorem 1. Then, we give more applications of the general result, dealing with $C^{2}$ diffeomorphisms of the two-disk with zero entropy having infinitely many periodic orbits.

Here are three examples in $C^{k}$-smooth dynamics with $k<\infty$ which together, serve as a general motivation to the present work. For simplicity, in this general introduction, we only care about $C^{k}$ when $k$ is an integer, which leaves aside many interesting questions! 
Example 1 by A. Denjoy. The so-called "Denjoy counterexamples" [De], first discovered by P. Bohl [Bo], are $C^{1}$-diffeomorphisms of the circle which have an irrational rotation number but no dense orbit.

Status of Example 1. Optimal. Another theorem by A. Denjoy [De] tells us that $C^{2}$ diffeomorphisms with topological dynamics similar to the above do not exist.

Example 2 by J. Harisson. The Seifert Conjecture asserts that every vector field on the three sphere has either a critical point or a periodic orbit. P. Schweitzer [Sc] used Example 1 to get $C^{1}$-counterexamples to the Seifert Conjecture, and J. Harisson later constructed $C^{2}$-counterexamples [Hj].

Status of Example 2. Unknown. One does not know if there exists a $C^{3}$-counterexample to the Seifert Conjecture.

Example 3 by J. Franks and L.S. Young. In [BF], R. Bowen and J. Franks constructed a $C^{1}$-smooth embedding of the 2-disk with:

(i) zero entropy,

(ii) one periodic orbit with period $2^{n}$ for each $n \geq 0$ and no other periodic orbit,

(iii) a single invariant Cantor set,

(iv) a non-wandering set completely described by (ii) and (iii).

Recall that a point $P$ is non-wandering for the map $F$ if for any neighborhood $U$ of $P$, there is a positive $n$ such that $F^{n}(U) \cap U \neq \emptyset$. The set of non-wandering points of $F$ is the non-wandering set of $F$; it is denoted by $\Omega(F)$.

Because of the status of Denjoy's counterexamples (also recall that in 1976, the best result about the Seifert Conjecture had the very same smoothness as given by Denjoy's theory), the question arose whether the Bowen-Franks example also had optimal smoothness. Then J. Franks and L.S. Young constructed a $C^{2}$ example satisfying (i), (ii), (iii) and (iv). It is the main new idea of this later method that we plan to extend to any dimension $d \geq 2$, so that when $d$ increases, we get smoother and smoother "challengers" to the real analytic $d$-dimensional examples adapted from [GST]. This new idea in [FY] consists in performing two period doubling bifurcations in the first approximation to the map they construct (it will become clear why this trick is so helpful).

The examples in $[\mathrm{BF}]$ and $[\mathrm{FY}]$, as well as the last ones we shall construct in Part I, do have all their periodic points hyperbolic. Some of these examples are built with the Kupka-Smale property, which means that all periodic orbits are hyperbolic with transversality of any pair of invariant manifolds of periodic points. Since all examples naturally carry a filtration corresponding to the hierarchical method of construction, the transversality part of the Kupka-Smale property, if not built in, is always easily obtained by local surgeries which are arbitrarily smooth (see below and [GST]).

Status of Example 3. Not Optimal. One knows [GST] that there exist real analytic embeddings of the 2-disk satisfying (i), (ii), (iii) and (iv). The method in [GST] is not quite constructive, and it seems reasonable to believe that the best one can do constructively is $C^{3-\varepsilon}$, but in Part I, we shall show that the method of Franks and Young gives constructive examples of increasing smoothness when working in higher and higher dimensional balls instead of the 2-disk. 


\section{Rigidity, Smoothness and Dimension}

\section{A. Introduction and Statement of the Result}

In this first part of the paper, we describe a simple method which yield $C^{1+\lfloor\sqrt{d}\rfloor}$. smooth embeddings of the $d$-dimensional ball with $d \geq 2$ with:

(i) zero entropy,

(ii) one periodic orbit of saddle type with period $2^{n}$ for each $n \geq 0$ and no other periodic orbit,

(iii) a single invariant Cantor set, which contains a small affine copy of itself, scaled by any ratio chosen from an interval,

(iv) a non-wandering set completely described by (ii) and (iii).

We use the notation $\lfloor x\rfloor$ for the integer part of $x$, and we notice that $1+\lfloor\sqrt{d}\rfloor$ is not an optimum choice for the smoothness. We choose this as an example of an increasing function $r(d)$ which goes to infinity with $d$ : this divergence is for us the main new observation and $1+\lfloor\sqrt{d}\rfloor$ allows the construction to be as simple as possible. We will also briefly indicate how $1+\lfloor\sqrt{d}\rfloor$ can be replaced by $r(d)=d+1-\varepsilon$, where $\varepsilon$ is an arbitrary small positive number.

The main point is that all the Cantor sets we shall construct will have a simple kind of scale invariance. In fact, generalizing to $d \geq 2$ the construction presented in [GST] (i.e. by perturbing to a diffeomorphism of the $d$-dimensional ball the singular fixed point of renormalization constructed by P. Collet, J.-P. Eckmann and H. Koch [CEK]) one gets embeddings of the $d$-ball satisfying (i), (ii), (iii) and (iv) which are real analytic. However the invariant Cantor sets constructed this way have a complicated geometry, inherited from the complicated scaling function of the one dimensional quadratic-like fixed point.

The existence of the simpler, hence "exotic," examples constructed here, means that one generally needs arbitrarily high smoothness to hope for complete rigidity, or global universality, of the cascade of period doubling bifurcations. This simple observation suggests a new perspective on the theory of dynamical rigidity.

The already mentioned works by Harisson [Hj] and Franks-Young [FY] and the one by G.R. Hall [Hg] give examples of papers concerned with the question of relationship between smoothness and dimension (see in particular the introduction sections of these papers). Clearly the idea that:

\section{"there are relations between the optimal smoothness of some examples in dynamics and dimension",}

is not new. The new point of view that we like to convey here is that the recent concept of Mostow-like dynamical rigidity [Su], called universality in physics [CEK,CT,Fe], should play a crucial role in this central question of smooth dynamics. A naive (and still imprecise) conjecture is that rigidity (accompanied by complicated scaling geometry) prevails in any dimension when the smoothness is so high that self-similar constructions cannot anymore be carried out: see [Tr] for related results in one dimension.

We now state the main result of Part I:

Theorem 1. For any $k>0$, there exists a small $\gamma>0$, a $d \geq 2$ and a $C^{k}$-smooth Kupka-Smale embedding of the d-dimensional ball with: 
(i) zero entropy,

(ii) one periodic orbit with period $2^{n}$ for each $n \geq 0$, which is a saddle, and no other periodic orbit,

(iii) a single invariant Cantor set which contains a small affine copy of itself, scaled by any factor in the interval $] \frac{1-2 \cdot \gamma}{1+2 \sqrt{d}}, \frac{1-\gamma}{1+2 \sqrt{d}}[$, for any small $\gamma>0$,

(iv) a non-wandering set completely described by (ii) and (iii).

Remark. These embeddings will be constructed as solutions of the functional equation:

$$
G=\Lambda^{-1} \circ G^{2^{d}} \circ \Lambda
$$

where $\Lambda$ is an affine transformation with norm:

$$
\nu \in] \frac{1-2 \cdot \gamma}{1+2 \cdot \sqrt{d}}, \frac{1-\gamma}{1+2 \cdot \sqrt{d}}[
$$

\section{B. Proof of Theorem 1}

To simplify the presentation, we first construct diffeomorphisms which badly fail to be Kupka-Smale: they have continua of periodic orbits which form shells (i.e. complements of $d$-balls in larger concentric $d$-balls), and act as translations on these shells. The construction of these degenerate examples will be carried out in three steps. In the fourth step, the non-hyperbolic periodic points will be removed as we will transform the degenerate diffeomorphisms to Kupka-Smale embeddings. Altogether, the proof has two main ingredients: a sequence of simple geometric constructions and a pair of obvious and well known, but quite powerful lemmas, which we call the Isotopy Cutting Lemma and the Norms Rescaling Lemma. These lemmas give us the control of the smoothness we need and play also a central role in [GT].

Step 1. The first approximation. Let $D^{d}$ stand for the $d$-dimensional unit ball in $\mathbf{R}^{d}$ for $d \geq 2$, and $D^{\prime d}$ for the concentric ball with radius $1-\gamma$, so that the complement of $D^{\prime d}$ in $D^{d}$ in a thin spherical shell $A_{0}$ with radius $\gamma$. The $d$ coordinate hyperplanes split the complement of their union in $D^{\prime d}$, into $2^{d}$ disjoint open regions $D_{0 ; i}^{d}, 0 \leq 1 \leq 2^{d}-1$. Each of these regions contains a closed ball with radius:

$$
\left.r_{0} \in\right] \frac{1-2 \cdot \gamma}{1+2 \cdot \sqrt{d}}, \frac{1-\gamma}{1+2 \cdot \sqrt{d}}[
$$

and there is enough room left to move rigidly any par of these balls, until they exchange their positions, without touching the other balls, see Fig. 1.

We then define $F_{0}$ as any diffeomorphism of $D^{d}$ which:

- preserves the boundary $\partial D^{d}$,

- is the identity in the shell $A_{0}$,

- exchanges by translations the $2^{d}$ balls $D_{0 ; i}^{d}$,

- is a Morse-Smale diffeomorphism in $M_{0}=D^{d} \backslash\left\{A_{0} \cup \bigcup_{0 \leq i<2^{d}-1} D_{0 ; i}^{d}\right\}$, with exactly one periodic orbit with period $2^{n}$ for each $n$ such $0 \leq n \leq d$ and no other periodic 
A d-dimensional

Fig. 1

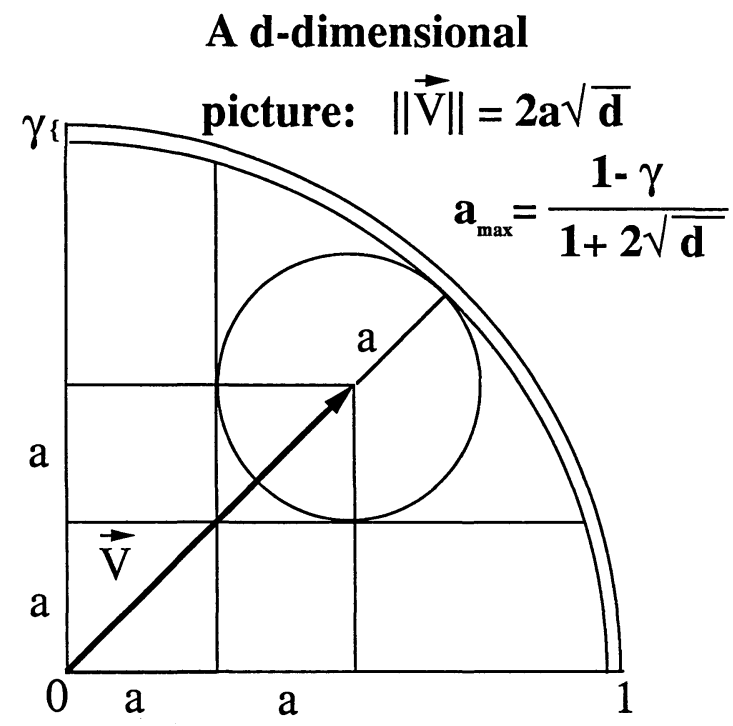

orbit in $M_{0}$. Furthermore, each of the periodic orbits is a saddle with a single unstable direction.

- the set $\bigcup_{0 \leq i<2^{d}-1} D_{0 ; i}^{d}$ is an attractor for $F_{0}$, while the shell $A_{0}$ is an attractor for the inverse map $F_{0}^{-1}$.

Nothing prevents $F_{0}$ from being constructed as a $C^{\infty}$ diffeomorphism. An isotopy from $F_{0}$ to the identity map Id acting on $D^{d}$ is realized by a torus flow $\left\{\Psi^{t}\right\}_{0 \leq t \leq 1}$ which suspends $F_{0}$ in the solid torus $D^{d} \times S^{1}$. We choose this suspension so that for all $t$ with $0 \leq t \leq 1, \Psi^{t}$ maps rigidly the $D_{0 ; i}^{d}$ 's and leaves the shell $A_{0}$ pointwise invariant. We write $F_{0}$ as the composition:

$$
F_{0}=F_{0 ; 2^{d}-1} \circ F_{0 ; 2^{d}-2} \circ \ldots \circ F_{0 ; 0}
$$

where $F_{0 ; i}$ is the restriction of the map $\Psi^{1 / 2^{d}}$ defined by:

$$
F_{0 ; i}=\left.\Psi^{1 / 2^{d}}\right|_{D \times\left\{i / 2^{d}\right\}}
$$

The choice of the radius $r_{0}$ in $] \frac{1-2 \cdot \gamma}{1+2 \cdot \sqrt{d}}, \frac{1-\gamma}{1+2 \cdot \sqrt{d}}[$ corresponds to the following
two imperatives: $-r_{0}$ has to be small enough so that the isotopy moves the disks $D_{0 ; i}^{d}$ rigidly for all $d \geq 2$,

- $r_{0}$ has to be large enough to allow a good control of the $C^{1+\lfloor\sqrt{d}\rfloor}$ norms in the construction of our model.

We postpone to the last paragraph of Part I, where we improve $r(d)$ 's value, a few hints on the way to improve the value of $r(d)$ up to $d+1-\varepsilon$ for any $\varepsilon>0$.

$F_{0}$ is represented in Fig. 2 in the cases when $d=2$ and $d=3$. 
Fig. 2

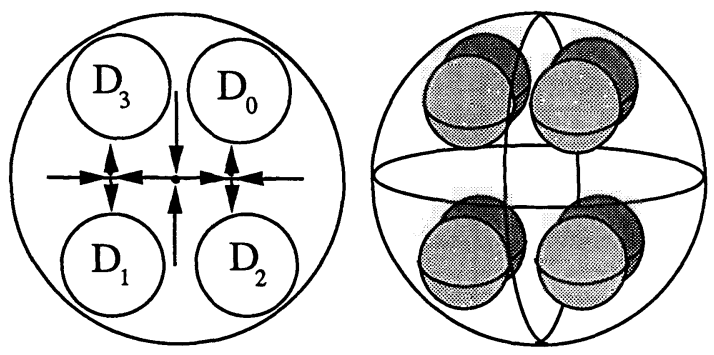

Step 2. Renormalization. Let $T_{i}$ be the translation which carries $D_{0 ; 0}^{d}$ to $D_{0 ; i}^{d}, R_{0}$ be an affine dilation which carries $D_{0 ; 0}^{d}$ to $D^{d}$ and let us write $R_{i}=R_{0} \circ T_{i}^{-1}$. We define $F_{1}$ by:

- $F_{1}(P)=F_{0}(P)$ if $P$ is in $D$ and not in a $D_{0 ; i}^{d}$,

- $F_{1}(P)=R_{(i+1) \bmod .2^{d}}^{-1} \circ F_{0 ; i} \circ R_{i}(P)$ if $P$ is in $D_{0 ; i}^{d}$.

This new map $F_{1}$ is again a $C^{\infty}$ diffeomorphism and has the three following important properties:

1) $F_{1}$ has $2^{2 d}$ balls with the same radius $r_{1}=\left(r_{0}\right)^{2}$ which are exchanged by translations. We denote these disks $D_{1 ; i, j}^{d}$, where $0 \leq i, j<2^{d}-1$. Here $i$ means that $D_{1 ; i, j}^{d}$ belongs to $D_{0 ; i}^{d}$. More precisely, $D_{1 ; i, j}^{d}$ belongs to the complement in $D_{0 ; i}^{d}$ of a shell $A_{1 ; i}^{d}$ with radius $\frac{\mu}{2^{d}}$ such that $\partial D_{0 ; i}^{d}$ is also the exterior boundary of $A_{1 ; i}^{d}$. Furthermore:

$$
F_{1}\left(D_{1 ; i, j}^{d}\right)=D_{1 ;(i+1) \bmod .2^{d},(j+1) \bmod .2^{d}}^{d}
$$

and $F_{1}$ acts on each shell $A_{1 ; i}^{d}$ like a translation.

2) $F_{1}$ is a Morse-Smale diffeomorphism in

$$
M_{1}=D^{d} \backslash\left\{A \cup \bigcup_{0 \leq i<2^{d}-1} A_{1 ; i}^{d} \cup \underset{0 \leq i<2^{d}-1,0 \leq j<2^{d}-1}{\bigcup} D_{1 ; i, j}^{d}\right\}
$$

with exactly one periodic orbit with period $2^{n}$ for each $n$ such $0 \leq n \leq 2^{d}-1$ and no other periodic orbit in $M_{1}$. Furthermore, each of the periodic orbits is a saddle with a single unstable direction.

3) The set $0 \leq i<2^{d}-1,0 \leq j<2^{d}-1$

$D_{1 ; i, j}^{d}$ is an attractor for $F_{1}$, the shell $A_{0}$ is an attractor for the inverse map $F_{1}^{-1}$, and the set $\bigcup_{0 \leq i<2^{d}-1} A_{1 ; i}^{d}$ is an attractor for the restriction of $F_{1}$ to $M_{0}$, but an attractor for the restriction of the inverse map $F_{1}^{-1}$ to $\bigcup_{0 \leq i<2^{d}-1} D_{0 ; i}^{d}$.

A construction similar to the one giving $F_{1}$ in the cases when $d=2$, is represented in Fig. 2.

More generally, assume we have constructed $F_{n}$ which has $\left(2^{d}\right)^{n}$ balls $D_{n ; i_{0}, i_{1}, \ldots, i_{n}}^{d}$ exchanged by translations according to:

$$
F_{n}\left(D_{n ; 2_{0}, i_{1}, \ldots, i_{n}}^{d}\right)=D_{n ;\left(i_{0}+1\right) \bmod .2^{d}, \ldots,\left(i_{n}+1\right) \bmod .2^{d}}^{d}
$$


Then we set:

$$
g\left(n ; i_{0}, i_{1}, \ldots, i_{n}\right)=i_{0}+\left(2^{d}\right) \cdot i_{1}+\ldots+\left(2^{d}\right)^{n-1} \cdot i_{n}
$$

and:

$$
F_{n ; i_{0}, i_{1}, \ldots, i_{n}}=\left.\Psi^{1 /\left(2^{d}\right)^{n}}\right|_{D^{d} \times\left\{g\left(n ; i_{0}, i_{1}, \ldots, i_{n}\right) /\left(2^{d}\right)^{n}\right\}}
$$

we denote:

- $T_{g\left(n ; i_{0}, i_{1}, \ldots, i_{n}\right)}$ the translation which carries $\dot{D}_{n ; 0,0, \ldots, 0}^{d}$ to $D_{n ; i_{0}, i_{1}, \ldots, i_{n}}^{d}$ and:

$-R_{n ; 0,0, \ldots, 0}$ the affine dilation which carries $D_{n ; 0,0, \ldots, 0}^{d}$ to $D^{d}$, and we write:

$$
R_{g\left(n ; i_{0}, i_{1}, \ldots, i_{n}\right)}=R_{n ; 0,0, \ldots, 0} \circ T_{g\left(n ; i_{0}, i_{1}, \ldots, i_{n}\right)}^{-1} .
$$

This allows us to simply define $F_{n+1}$ by:

- $F_{n+1}(P)=F_{n}(P)$ if $P$ is in $D$ and not in a $D_{n ; i_{0}, i_{1}, \ldots, i_{n}}^{d}$,

- $F_{n+1}(P)=R_{\left(g\left(n ; i_{0}, i_{1}, \ldots, i_{n}\right)+1\right) \bmod .\left(2^{d}\right)^{(n+1)}}^{-1} \circ F_{n ; i_{0}, i_{1}, \ldots, i_{n}} \circ R_{g\left(n ; i_{0}, i_{1}, \ldots, i_{n}\right)}(P)$ if $P$ is in $D_{0 ; i}^{d}$.

For each $m$, the map $F_{m}$ constructed by this inductive process is again a $C^{\infty}$ diffeomorphism: the shell $A_{0}$ and its successive reduced copies $A_{n ; i_{0}, i_{1}, \ldots, i_{n}}^{d}$ allow the surgery which transforms $F_{m-1}$ into $F_{m}$ to be arbitrarily smooth. Furthermore, for each $m$, let us define the set:

$$
\begin{aligned}
M_{m}= & D^{d} \backslash\left\{A_{0} \cup \bigcup_{0 \leq i<2^{d}-1} A_{1 ; i}^{d} \cup \ldots\right. \\
& \left.\ldots \cup \cup \bigcup_{\Pi 0 \leq i_{k}<2^{d}-1} A_{n ; i_{0}, i_{1}, \ldots, i_{n-1}}^{d} \cup \underset{\Pi 0 \leq i_{k}<2^{d}-1}{\bigcup} D_{n ; i_{0}, i_{1}, \ldots, i_{n}}^{d}\right\} .
\end{aligned}
$$

Then the map $F_{m}$ restricted to $M_{m}$ is a Morse-Smale diffeomorphism with exactly one hyperbolic periodic orbit with period $2^{n}$ for each $n$ such $0 \leq n \leq(m+1) \cdot d-1$ and no other periodic orbit. Again:

- Each of the periodic orbits is a saddle with a single unstable direction.

- The set $\bigcup D_{n ; i_{0}, \imath_{1}, \ldots, i_{n}}^{d}$ is an attractor for $F_{m}$, the shell $A_{0}$ is an attractor $\Pi 0 \leq i_{k}<2^{d}-1$

for the inverse map $F_{m}^{-1}$ and each of the sets of the form $\bigcup_{\prod 0 \leq i_{k}<2^{d}-1} A_{n ; i_{0}, i_{1}, \ldots, i_{n-p}}^{d}$ is an attractor for the restriction of $F_{m}$ to $M_{m}$, as well as an attractor for the restriction of the inverse map $F_{m}^{-1}$ to $\bigcup_{\prod 0 \leq i_{k}<2^{d}-1}^{m} D_{n ; i_{0}, i_{1}, \ldots, l_{n-p-1}}^{d}$.

The next thing we want to understand is the smoothness of the map $F$ obtained as the limit of the $F_{m}$ 's.

Step 3. Limit and Control of the Smoothness. The sequence $\left\{F_{m}\right\}$ is made of $C^{\infty}$ diffeomorphisms, but has no chance to converge in the $C^{\infty}$ topology. The problem is of course on the small scales, where $F_{p}$ differs from $F_{p+1}$ as $p$ becomes unbounded.

However: 
Claim.

$$
\left\{F_{m}\right\} \text { is a Cauchy sequence in the } C^{1+\lfloor\sqrt{d}\rfloor} \text { topology. }
$$

This claim insures that our example (the limit $F$ of $\left\{F_{m}\right\}$ in the $C^{1+\lfloor\sqrt{d}\rfloor}$ topology) is a $C^{1+\lfloor\sqrt{d}\rfloor}$ diffeomorphism of the disk. Assuming that the Claim is true, to prove Theorem 1, it only remains to get rid of the degenerate orbits and to treat the transversality of the invariant manifold crossings. Hence we postpone the proof of the claim which is the only small analysis part.

Step 4. Kupka-Smale Embeddings with the Right Non-Wandering Set. When $d=2$, an easy modification of our construction yields Kupka-Smale diffeomorphisms with properties (i), (ii) and (iii): it is enough to change $F_{0}$ by imposing a rigid rotation with irrational rotation number $\theta$ in the annulus $A_{0}$, and by imposing a rigid rotation with irrational rotation number $\frac{\theta}{4}$ in each of the 4 disks $D_{0 ; 2}^{2}$. These changes then propagate by self-similarity, using obvious modifications of the isotopies, but all this is not enough to give us property (iv) of the theorem.

A naive generalization to arbitrary dimensions of the same modifications would not even yield Kupka-Smale diffeomorphisms when $d \neq 2$, but we shall present a two point procedure working for all $d \geq 2$.

Remark. Each of the following points should be accompanied by a modification of the flow $\Psi^{t}$ which yields the isotopy, whose detailed construction is left to the reader.

Point 1 . We change $F_{0}$ to $F_{0}^{\prime}$ by modifying $F_{0}$ :

- first in the neighborhood of a shell $A_{0}$, so that $D$ be mapped inside itself (recall that we had arranged the shell $A_{0}$ to be a repeller),

- then in the neighborhood of $\bigcup_{0 \leq i<2^{d}-1} D_{0 ; i}^{d}$, so that the image of $M_{0}$ is not contained in itself, but in $M_{0} \cup \bigcup_{0 \leq i<2^{d}-1} A_{1 ; i}^{d}$ (recall that we had arranged the shells $A_{1 ; i}^{d}$ to be attractors on one side, and repellers on the other side).

These changes then propagate by self-similarity. At this stage we have gotten rid of all degenerate periodic points, but might have introduced heteroclinic tangencies.

Notice that these modifications of the map $F_{0}$ to $F_{0}^{\prime}$ could be made arbitrarily small in the $C^{\infty}$ topology: radially for $A_{0}$ in $D^{d}$ and for $A_{1 ; i}^{d}$ in $D_{0 ; i}^{d}$, this corresponds to modifications as illustrated in Fig. 3.

Point 2. We change $F_{0}^{\prime}$ to $F_{\theta}^{\prime \prime}$ by suppressing all possible non-transversality of the invariant manifolds of the periodic points. Of course, no tangency was present when starting with $F_{0}$, since the invariant shells at each stage isolated successive hierarchical levels of the construction. Since we are left with a filtration after Point 1 , the situation is easily handled by suppressing any degeneracy successively at finer and finer levels of the construction. The filtration insures that the only tangencies which can occur are between the unstable manifold of a $2^{n}$-cycle with the stable manifold of a $2^{m}$-cycle with $m>n$.

The fact that one has to work at finer and finer scales is not an obstacle to whatever smoothness we like: in order to suppress a degeneracy, the change, which is performed in a fundamental domain, can be made as small as one likes. If we choose these surgeries as being e.g. more than exponentially smaller when working 

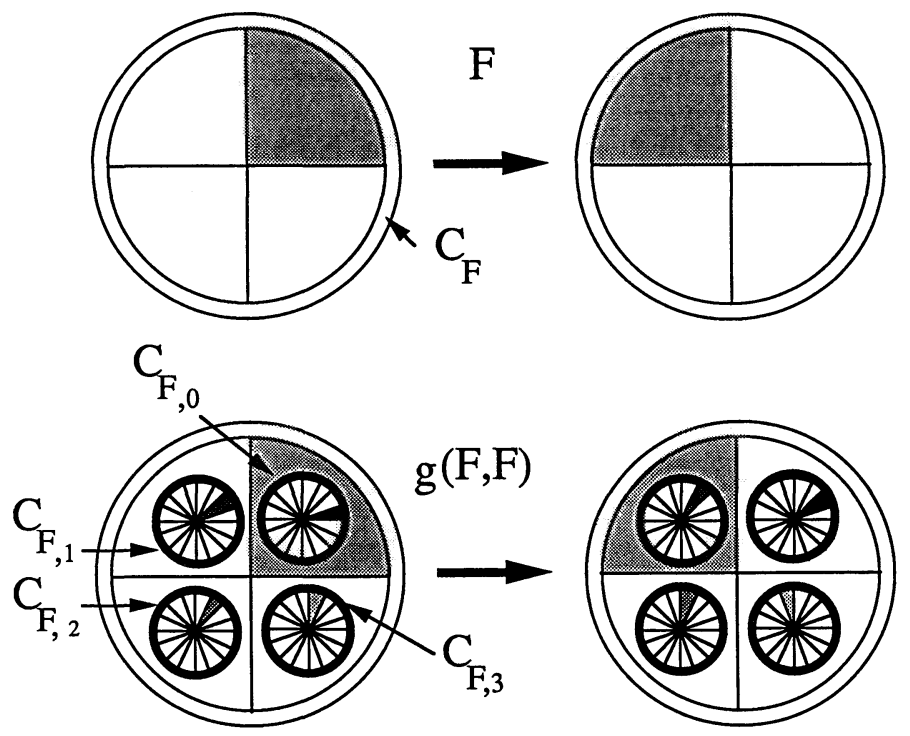

Fig. 3

at the exponentially finer successive scales of the construction, the Norms Rescaling Lemma insures that we do not loose any of our control on the regularity.

We shall refer to the construction described in Points 1 and 2 as the Cleaning Construction.

$\square$ (Theorem 1).

Proof of the Claim. The truth of the claim itself depends on the fact that in the $C^{1+\lfloor\sqrt{d}\rfloor}$ norm, $\|\cdot\|_{1+\lfloor\sqrt{d}\rfloor},\left\|F_{m+1}-F_{m}\right\|_{1+\lfloor\sqrt{d}\rfloor}$ decreases as $C \cdot \Gamma^{m}$ for some positive constant $C$, where $\Gamma=\varrho^{\alpha_{0}}<1$, with $\alpha_{0}=\frac{-\lfloor\sqrt{d}\rfloor \cdot \log (\varrho)+d \cdot \log 2}{\varrho}$, $\varrho=\frac{1-2 \cdot \gamma+\delta}{1+2 \sqrt{d}}$ and $\delta<\gamma$.

Of course, all the contribution to $\left\|F_{m+1}-F_{m}\right\|_{1+\lfloor\sqrt{d}\rfloor}$ comes from the balls $D_{m ; i_{0}, i_{1}, \ldots, i_{m}}$, since this is the only place where the two maps differ (more precisely the two maps differ in the complement, in these disks, of the small copies of the shell $\left.A_{0}\right)$.

Up to the translations $T_{g\left(m ; i_{0}, i_{1}, \ldots, i_{m}\right)}$ and $T_{\left(g\left(m ; i_{0}, i_{1}, \ldots, i_{m}\right)+1\right) \text { mod.4m+1 }}^{-1}$ (which does not contribute to $\|\cdot\|_{r}$ for $r>1$ ), the restriction of $F_{m+1}$ to $D_{m ; i_{0}, i_{1}, \ldots, i_{m}}$ is just a rescaled copy of $\left.\Psi^{1 / 4^{m}}\right|_{D \times\left\{g\left(m ; i_{0}, \imath_{1}, \ldots, i_{m}\right) / 4^{m}\right\}}$. It follows that the exponential decay $C \cdot \gamma^{m}$ of $\left\|F_{m+1}-F_{m}\right\|_{1+\lfloor\sqrt{d}\rfloor}$ is a simple consequence of the two following elementary but fundamental lemmas:

Isotopy Cutting Lemma. Let $G$ be a $C^{r}$ diffeomorphism of the ball and $G_{t}$ an isotopy going from the identity $G_{0}$ to $G_{1}=G$. Then there exists a constant $K>0$ such that, for all $N>0$ and $0 \leq i<N$ :

$$
\left\|G_{(i+1) / n} \circ\left(G_{i / N}\right)^{-1}-\mathrm{Id}\right\|_{r}<K / N .
$$

Norms Rescaling Lemma. Let $G$ be a $C^{r}$ diffeomorphism of the unit ball $D$, and $\Lambda$ be an affine map sending another (not necessarily round) ball $D^{\prime}$ with radius $\varrho$ to $D$. 
Then for any integer $k \leq r$ :

$$
\left\|\Lambda^{-1} \circ D^{(k)} G \circ \Lambda\right\|_{0} \leq\left\|D^{(k)} G\right\|_{0} \cdot \varrho^{1-k} .
$$

In our problem, when $m$ is large enough, the Norms Rescaling Lemma implies that $\left\|F_{m}-\operatorname{Id}\right\|_{1+\lfloor\sqrt{d}\rfloor}=\left\|D^{(1+\lfloor\sqrt{d}\rfloor)}\left(F_{m}\right)\right\|_{0}$. Consequently, when estimating the norm $\left\|F_{m+1}-F_{m}\right\|_{1+\lfloor\sqrt{d}\rfloor}$, the Isotopy Cutting Lemma gives us:

$$
\left\|F_{m ; i_{0}, i_{1}, \ldots, i_{m}}-\operatorname{Id}\right\|_{1+\lfloor\sqrt{d}\rfloor} \leq \frac{K}{\left(2^{d}\right)^{m}} .
$$

The Norms Rescaling Lemma then implies:

$$
\left\|F_{m+1}-F_{m}\right\|_{1+\lfloor\sqrt{d}\rfloor} \leq \frac{K}{\left(2^{d}\right)^{m} \cdot\left(\frac{1-2 \cdot \gamma+\delta}{1+2 \sqrt{d}}\right)^{m \cdot\lfloor\sqrt{d}\rfloor}}
$$

About the Lemmas. Both lemmas are already implicit in [RT], and might as well be older. The Isotopy Cutting Lemma (without the name!) is stated in a particular case in [FY], together with a proof, which, up to a trivial rewording, covers all our needs. The Norms Rescaling Lemma is a trivial computation hidden e.g. in the $\|\cdot\|_{2}$ estimates of [FY]. Our only contribution is to have isolated these statements. They are quite useful:

- as powerful tools for self similar constructions,

- as simple guides in the approach to rigidity ideas.

Let us mention two ways to understand the Norms Rescaling Lemma:

$\alpha$ - affine self-similarity does not go along well with high smoothness,

$\beta$ - topological self similarity might need complicated metric rescalings to be realized by very smooth maps.

For other applications, see also [GT] and the next part of the present paper.

Improving $r(d)$ 's Value.

1. From standard estimates, it follows that $F$ is indeed slightly more regular that just $C^{1+\lfloor\sqrt{d}\rfloor}$. More precisely $F$ is of class $C^{1+\lfloor\sqrt{d}\rfloor+\alpha}$ for any $\alpha$ such that $0 \leq \alpha<\alpha_{0}$, and we notice that $\alpha$ is greater than one when $d$ is large.

2. To obtain $r(d)=d+1-\varepsilon$ for all $\varepsilon$ such that $\varepsilon>0$, instead of the $r(d)=1+\lfloor\sqrt{d}\rfloor$ we had gotten so far, it is enough to systematize the way [FY] improved on $[\mathrm{BF}]$ : more precisely, it is enough to incorporate more and more period doubling bifurcations at the level of the first approximation $F_{0}$ to get closer and closer to $d+1$ which stays unreachable by such surgical attempts. The computation goes as follows:

- Combining the Isotopy Cutting Lemma and the Norms Rescaling Lemma, one would need $n$ balls with radius $\varrho$ such that $n \cdot \varrho^{d}>1$ in order to get an $F$ of class $C^{d+1}$, but the same $n \cdot \varrho^{d}>1$ just means that there is not enough room in the unit ball.

- In order to obtain $C^{d+1-\varepsilon}$, it is easier to first remark that the Norms Rescaling Lemma can be used as well for fractional powers. Then, in order to get an $F$ of class $C^{d+1-\varepsilon}$, we just need the number and radius to satisfy $n \cdot \varrho^{d-\varepsilon}>1$, and a system of identical balls satisfying this last inequality can well fit inside the unit ball if $n$ is chosen to be large enough. 


\section{A General Result and Some Applications}

\section{A. A General Result}

There are many results similar to Theorem 1 . In order to avoid more repetitions, we formalize a general procedure which follows the steps of the proof of Theorem 1 .

Let $Z$ be a set of indices and let the set of 1-models $\left\{F_{z}\right\}_{z \in Z}$ be a collection of $C^{\infty}$ diffeomorphisms of $D^{d}$, isotopic to the identity map $\operatorname{Id}_{D^{d}}$ on $D^{d}$, and such that: - $D^{d}$ contains $n \equiv n(z)$ small identical disjoint closed $d$-balls $\left\{D_{z, j}^{d}\right\}_{j \in\{0,1, \ldots, n-1\}}$ with:

$$
\begin{aligned}
& -F_{z}\left(D_{z, j}^{d}\right)=D_{z,(j+1) \bmod . n}^{d}(F), \\
& -\left.F_{z}\right|_{D_{z, j}^{d}\left(F_{z}\right)} \text { is an isometry. }
\end{aligned}
$$

- there are tubular neighborhoods:

$$
-F_{z} \text { of } \partial D^{d} \text { in } D^{d} \backslash\left\{\bigcup_{0 \leq j<n-1} D_{z, j}^{d}\right\}
$$

and:

- $C_{z, j}$ of each $\partial D_{z, j}^{d}$ in $D^{d} \backslash\left\{C_{z} \cup \bigcup_{0 \leq j<n-1} D_{z, j}^{d}\right\}$

such that:

- $\left.F\right|_{C_{z}}$ and $\left.F\right|_{C_{z, j}}$ are isometries of $C_{z}$ and of the $C_{z, j}$ 's, with $F\left(C_{z}\right)=C_{z}$, $F\left(C_{z, j}\right)=C_{z(j+1) \bmod . n}$,

and:

- $C_{z}$ is a repeller, and the $C_{z, j}$ 's are attractors, at least marginally.

An isotopy from $F$ to the identity map $\operatorname{Id}_{D^{d}}$ is realized by any suspension $\left\{\Psi_{z}^{t}\right\}_{0 \leq t \leq 1}$ of $F_{z}$ in $D^{d} \times S^{1}$. We choose this suspension so that for all $t$ with $0 \leq t \leq 1, \Psi_{z}^{t}$ maps isometrically $C_{z}$ and the $D_{z, j}$ 's. For any $m>0$, we write $F_{z}$ as the composition:

$$
F_{z}=F_{z, m-1} \circ F_{z, m-2} \circ \ldots \circ F_{z, 0},
$$

where $F_{z, i}$ is the restriction of the map $\Psi_{z}^{1 / m}$ defined by:

$$
F_{z, i}=\left.\Psi_{z}^{1 / m}\right|_{D \times\{i / m\}}
$$

Now choose any pair $\left(z_{0}, z_{1}\right)$, and let $\mathbf{R}_{z_{0}, \imath}$ be an affine dilation which carries $D_{z_{0}, i}^{d}$ to $D^{d}$.

This allows us to simply define $g_{z_{0}, z_{1}}=g\left(F_{z_{0}}, F_{z_{1}}\right)$ by:

- $g_{z_{0}, z_{1}}(P)=F_{z_{0}}(P)$ if $P$ is in $D^{d}$ and not in a $D_{z_{0}, i}^{d}$,

$-g_{z_{0}, z_{1}}(P)=\mathbf{R}_{z_{0},(i+1) \bmod . n}^{-1} \circ F_{z_{1}, i} \circ \mathbf{R}_{z_{0}, i}(P)$ if $P$ is in $D_{z_{0}, i}^{d}$.

The set of 2-models is by definition the set of $C^{\infty}$ diffeomorphisms of $D^{d}$ which can be constructed as $g_{z_{0}, z_{1}}$. They all are isotopic to the identity map $\operatorname{Id}_{D^{d}}$ on $D^{d}$. The isotopy can be realized by a suspension $\left\{\Psi_{g_{G, F}}^{t}\right\}_{0 \leq t \leq 1}$ of $g_{z_{0}, z_{1}}$ in $D^{d} \times S^{1}$; we choose this suspension so that for all $t$ with $0 \leq t \leq 1, \Psi_{g_{G, F}}^{t}$ maps isometrically $C_{z_{0}}$ and the $C_{z_{0}, j}$ 's as well as the small copies of $C_{z_{1}}$ and of the $C_{z_{1}, i}$ 's in the $C_{z_{0}, j}$ 's. Consequently, understanding the $g_{z_{0}, z_{1}}$ 's as 1-models glued in 1-models, one can 
make an obvious generalization of the construction yielding the $g_{z_{0}, z_{1}}$ 's, and define $g_{z_{0}, z_{1}, z_{2}}=g\left(F_{z_{0}}, g_{z_{1}, z_{2}}\right)$ as a 2-model glued in a 1-model. Continuing inductively, we obtain $m$-models for each $m>1$.

For each $m$, the maps $g_{z_{0}, \ldots, z_{m-1}}$ constructed by this inductive process is again a $C^{\infty}$ diffeomorphism: the tubular neighborhoods $C_{z_{0}}$ and their successive reduced analogs $C_{z_{0}, \ldots, z_{p-1}}$ at each scale $p$, allow the surgery which transforms $g_{z_{0}, \ldots, z_{m-1}}$ into $g_{z_{0}, \ldots, z_{m}}$ to be arbitrarily smooth.

The $C_{z_{0}, i}$ 's and the analog $C_{z_{0}, \ldots, z_{p-1}, j}$ 's at each scale $p$ give the room to perform the modifications corresponding to the Cleaning Construction (i.e. points 1 and 2 in Part I), if necessary. For each $m$, we define the set:

$$
\begin{array}{r}
\mathfrak{M}_{m}=M_{s_{m}} \backslash\left\{C_{F_{1}} \cup \bigcup_{i} C_{F_{1}, i} \cup \bigcup_{1<p<m} C_{g_{F_{p}, \ldots, F_{1}}, j} \cup\right. \\
\bigcup_{1<p<m-1} \bigcup_{j}\left(C_{g_{F_{p}, \ldots, F_{1}, j}} \cup \bigcup_{k} D_{k}\left(g_{F_{m}, \ldots, F_{1}}\right),\right.
\end{array}
$$

and anything defined there will remain unchanged by further steps of the construction.

Theorem 2. a. $\left\{g_{z_{0}, \ldots, z_{m}}\right\}_{m}$ is a Cauchy sequence in some $C^{k}$ topology, where $k \geq k_{\min }=1$ always olds.

b. $k_{\min }=1$ can be improved using the combination of the Isotopy Cutting Lemma, the Norms Rescaling Lemma and, if any, the decay of all the $C^{r}$ norms $\left\|F_{m}\right\|_{r}$ of $F_{m}$ as $m \rightarrow+\infty$.

c. If all the $C^{r}$ norms $\left\|F_{m}\right\|_{r}$ decay fast enough, then $k=+\infty$.

d. The optimal smoothness $k$ is not affected by the Cleaning Construction.

e. The non-wandering set $\Omega\left(F_{\infty}\right)$ of the limit map $F_{\infty}$ is made of the disjoint union of the pieces in the $\mathfrak{M}_{m}$ 's coming from the $\Omega\left(g_{z_{0}, \ldots, z_{m}}\right)$ 's, a set of degenerate periodic points at each level of gluing new pieces when passing from $g_{z_{0}, \ldots, z_{m-1}}$ to $g_{z_{0}, \ldots, z_{m}}$, and a Cantor set. The degenerate part is destroyed by the Cleaning Construction.

Theorem 2 is proven by obvious adaptations of the arguments used to prove Theorem 1. Details are left to the reader.

\section{B. Some Applications}

Our example in [GT] of a $C^{2}$ diffeomorphism of the two disk with infinitely many Axiom $A$ strange attractors is an application of Theorem 2 when all 1-models are a same diffeomorphism with an Axiom $A$ strange attractor and $n=4$. Here, we shall concentrate on maps with much simpler dynamics.

The periodic orbit structure of orientation preserving diffeomorphisms and embeddings of the two disk with zero topological entropy has been described in [GST]. Since, it has been asked whether infinite hierarchies of periodic orbits can coexist for zero entropy embeddings of the two disk which would be smooth enough., It seems to us that a complete answer to this question would involve much more theory than attainable now. We have 
Theorem 3. Let $\left\{\frac{p_{n}}{q_{n}}\right\}$ be any sequence of rational numbers written in reduced form with $\frac{p_{1}}{q_{1}}=\frac{0}{1}$. There exists a $C^{2}$ diffeomorphism of the two disk, $F$, with zero topological entropy, such that for all $n \geq 1$ :

- $F$ has a pair of periodic orbits $O_{n}=\left\{M_{1}, M_{2}, \ldots, M_{P_{n}}\right\}$ and $O_{n}^{\prime}$ with the same period $P_{n}=q_{1} \cdot q_{2} \cdot \ldots \cdot q_{n}$,

- $F^{P_{n}}$ splits $O_{n+1}$ in $P_{n}$ periodic orbits $\left\{O_{n, i}\right\}_{i \in 1, P_{n}}$ with period $q_{n+1}$, each having linking number $\frac{p_{n+1}}{q_{n+1}}$ with one point $M_{i}$ of $O_{n}$.

Theorem 4. There exists a Kupka-Smale diffeomorphism like in Theorem 1, with no other periodic orbit and such that for all $n \geq 1$ the periodic orbits $O_{n}$ is a sink.

Theorem $5[\mathrm{Ka}]$. The examples in Theorem 3 and in Theorem 4 can be made $C^{\infty}$ by letting $\frac{p_{n}}{q_{n}}$ go to zero fast enough when $n \rightarrow+\infty$.

Remark. Theorem 5 describes a situation, called "unbounded renormalization", which is the most difficult case, still misunderstood, in the case of endomorphisms of the interval or of the Riemann sphere.

From [GST] we also have:

Theorem 6 [GST]. The examples in Theorem 3 and in Theorem 4 can be made $C^{\infty}$ by letting $\frac{p_{n}}{q_{n}}$ be $\frac{1}{2}$ for $n$ large enough.

Hints for the Proofs of Theorems 3, 4, 5. If for all $n$ large enough, $q_{n}>3$, we associate a 1-model of $F$ to each $\frac{p_{n}}{q_{n}}$ in the form of the rigid rotation with angle $\frac{p_{n}}{q_{n}}$, modified in its $C_{F}$ and $C_{F, i}$ 's according to the $\frac{p_{n+1}}{q_{n+1}}$ one wants to plug in its $D_{i}(F)$ 's; these are quite degenerate maps that can we further modify to be Morse-Smale (i.e. Kupka-Smale with finitely many periodic orbits) out of $C_{F} \cup \cup_{i} C_{F, i}$. It then only remains to apply Theorem 2 (see Fig. 4 when $\frac{p_{n}}{q_{n}}=\frac{1}{4}$, for each $n$ ):

- First, Theorem 5 is quite simple: slow rotations are good approximations of the identity map, so that this result has been rediscovered over and over again. To our knowledge however, it first appeared in [Ka].

- Then, we remark that if $q_{n}>3, q_{n}$ small disks of radius greater than $\frac{1}{q_{n}}$ can fit in the unit disk, so that the combination of the Isotopy Cutting Lemma and the Norms Rescaling Lemma yields the $C^{2}$ result.

If infinitely often, we find a $q_{n} \leq 3$, we adapt the main idea of [FY] and introduce 1-models which combine to successive values $\frac{p_{n+1}}{q_{n+1}}$ and $\frac{p_{n}}{q_{n}}$, so that $q_{n+1} \cdot q_{n}$ small disks of radius greater than $\frac{1}{q_{n+1} \cdot q_{n}}$ can fit in the unit disk. Again, the combination of the Isotopy Cutting Lemma and the Norms Rescaling Lemma then yields the $C^{2}$ result. 
Acknowledgements. J.M.G. thanks I.B.M. France and the T.J. Watson Research Center where this work was carried out.

\section{References}

[BF] Bowen, R., Franks, J.: The periodic points of maps of the disk and the interval. Topology 15, 337-342 (1976)

[Bo] Bohl, P.: Über die hinsichtlich der unabhängigen und abhängigen Variablen periodische Differentialgleichung erster Ordnung, Acta Math. 40, 321-336 (1916)

[CEK] Collet, P., Eckmann, J.-P., Koch, H.: Periodic doubling bifurcations for families of maps on $R^{n}$. J. Stat. Phys. 25, 1-15 (1980)

[CT] Collet, P., Tresser, C.: Itérations d'endomorphismes et groupe de renormalisation. J. Phys. C5, 25-28 (1978), C.R. Acad. Sc. Paris 287A, 577-580 (1978)

[De] Denjoy, A.: Sur le courbes défines par les équations différentielles à la surface du tore. J. Math. Pures Appl. 11, 333-375 (1932)

[Fe] Feigenbaum, M.: The universal metric properties of non-linear transformations. J. Stat. Phys. 19, 25-52 (1978); 21, 7669-70 (1979)

[FY] Franks, J., Young, L.S.: A $C^{2}$ Kupka-Smale diffeomorphism of the disk with no source or sinks. In: Dynamical systems and turbulence Warwick 1980. Lecture Notes in Mathematics Vol. 898 Berlin, Heidelberg, New York: Springer

[GST] Gambaudo, J.M., van Strien, S., Tresser, C.: Hénon-like maps with strange attractors: there exists a $C^{\infty}$ Kupka-Smale diffeomorphism on $S^{2}$ with neither sinks nor sources. Nonlinearity 2, 287-304 (1989)

[GT] Gambaudo, J.M., Tresser, C.: Diffeomorphisms with infinitely many strange attractors. Journal of Complexity 6, 409-416 (1990)

[Hg] Hall, G.R.: Bifurcation of an attracting invariant circle: a Denjoy attractor. Ergodic Theory Dynam. Syst. 3, 87-118 (1983)

[Hj] Harisson, J.: $C^{2}$ Counterexamples to the Seifert conjecture. Topology 27, 249-278 (1988)

[Ka] Kan, I.: Strange attractors of uniform flows. Trans. Am. Math. Soc. 293, 135-159 (1986)

[RT] Ruelle, D., Takens, F.: On the nature of turbulence. Commun. Math. Phys. 20, 167-192 (1971), 23, 243-244 (1971)

[Sc] Schweitzer, P.: Counterexamples to the Seifert conjecture and opening leaves of foliations. Ann. Math. 100, 386-400 (1974)

[Su] Sullivan, D.: Bonds, quadratic differentials, and renormalization conjectures. To appear in A.M.S. Centenial Publication, Vol.2, Mathematics into the twenty-first Century, Providence: Am. Math. Soc.

[Tr] Tresser, C.: Fine structure of universal Cantor sets. In: Instabilities and nonequilibrium structures III. Tirapegui E., Zeller, W. (eds.) Dordrecht, Boston, Lancaster, Tokyo: Reidel 